\title{
A Further Study of the Effect of Insulin upon the Epinephrine Load of the Suprarenal Glands in Rabbits.
}

\author{
By \\ Zyumpei Kanowoka. \\ (鹿岡順平) \\ (From the Physiological Laboratory of Professor Y. Satake, \\ Tohoku Imperial University, Sendai.)
}

That insulin increases the liberation rate of epinephrine from the suprarenal gland and diminishes the load of epinephrine therein, is a well established fact.1) However there are some reports, the findings of which apparently do not harmonize well with the general view, above referred to. In the first place an unmistakable increase results, according to them, in the epinephrine load when insulin is administered repeatedly for a long interval of days, and secondly, the load is found to increase within a few minutes when insulin is given intravenously.

$\mathrm{N}$ o $\mathrm{sa} \mathrm{k} \mathrm{a}^{2}$ injected insulin in daily doses varying from 0.05 c.c. to 0.24 c.c. to rats for 12 days or so, and on the last day the animals were killed without receiving the hormone. For the control animals the physiological saline solution was applied in doses varying from 0.5 c.c. to 2 c.c. instead of insulin. The epinephrine was estimated by the colorimetrical method of Suto. The content of epinephrine in the insulin rats was found to be $0.01975 \mathrm{mgrm}$. $(0.01500$ $0.03000)$ in toto and $0.74 \mathrm{mgrm}$. per grm. glands $(0.59-0.90 \mathrm{mgrm}$.), while it was $0.01753 \mathrm{mgrm}$. $(0.01033-0.04500)$ in toto and $0.51 \mathrm{mgrm}$. per grm. $(0.39-0.83)$ in the control ones. The suprarenals were heavier in the latter; $0.0270 \mathrm{grm} .(0.273$ mgrm. per 100 grms. body wejght) in the former, and $0.0326 \mathrm{grm}$. $(0.253 \mathrm{mgrm}$. per 100 grms.) in the latter. The writer lays much stress upon the comparison of the epinephrine content reckoned on the gland weight.

Ohara ${ }^{3)}$ repeated similar experiments on rabbits and more extensively.

1) Saito, Tohoku J. of Exp. Med., 1929, 12, 263; Yen, A omura and Inaba, Ibid., 1933, 21, 542. Complete references there.

2) Nos a ka, Naibumpigaku Zassi, 1925(-6), 1, $647 \mathrm{ff}$.

3) O ha ra, Kumamoto Igakkai Zassi, 1928, 4, 898-905 (German abstract p. 66). 
From twenty to eighty days the subcutaneous injection of insulin in doses varying from about 0.2 to 2 units per kilo a day was given; in two cases the injection was given every day for about four hundred days. The time allowed to elapse between the last insulin and the killing was usually from 24 hours to 52 hours, only in two cases about twenty days. The epinephrine was estimated by the Suto method. In his experiments the normal rabbits, 8 in number, yielded $0.172 \mathrm{mgrm}$. (0.110-0.256 mgrm.) epinephrine in both glands, which corresponds to $0.044 \mathrm{mgrm}$. per grm. gland (0.0276-0.0635 mgrm.) and $0.083 \mathrm{mgrm}$. per kilo of body weight $(0.041-0.111 \mathrm{mgrm}$.), and the insulin rabbits, 18 in number, gave $0.243 \mathrm{mgrm}$. (0.077-0.438 mgrm.) epinephrine, which corresponds to $0.0411 \mathrm{mgrm}$. per grm. gland (0.0164-0.0707 mgrm.) and $0.098 \mathrm{mgrm}$. per kilo (0.031-0.193 mgrm.). The glands were enormously large in 4 individuals in the latter set of animals; they received insulin for $396,391,84$ and 36 days respectively. 5 rabbits, which received an inactive insulin solution every day for 28136 days, gave suprarenal capsules weighing similarly with the insulin rabbits and containing epinephrine in similar amounts to the normal rabbits. The values of epinephrine reckoned on the gland weight or the body weight were therefore decidedly smaller in the control animals, even compared with the normal individuals. The figures were $0.163 \mathrm{mgrm}$. $(0.122-0.213 \mathrm{mgrm}$.) in toto, $0.0264 \mathrm{mgrm}$. per grm. gland (0.0180-0.0334 mgrm.) and $0.068 \mathrm{mgrm}$. per kilo body weight $(0.044-0.091 \mathrm{mgrm}$.). By comparing such figures $\mathrm{O}$ ha r a concluded an increase of the epinephrine storage in the suprarenal bodies, which expresses, according to the writer, a compensatory hyperfunction of the suprarenals against the excess of the pancreas hormone.

Results contradictory to the above findings are seen in the communication of an Italian experimentalist. ${ }^{4}$ A moderate amount of insulin was repeatedly administered to dogs and guinea-pigs for 10-15 days, and 48 hours after the last injection the animals were killed, and no alteration was witnessed in the suprarenal capsules by examining them histologically.

A transitory increase of the epinephrine load of the suprarenal capsules was mentioned by $\mathrm{Hiraki}{ }^{5}$ ) on injecting the hormone intravenously to rabbits, contrary to the cases of subcutaneous application. A dose of 1 unit insulin per kilo of body weight, given intravenously, effected no alteration at all in the epinephrine content of the suprarenals. But 2 units were capable of causing an increase of the epinephrine load 2-5 minutes after the injection, afterwards it soon inclined to diminish. $0.8-1.3 \mathrm{mgrm}$. epinephrine was measured in every gland, which corresponds to 0.5-1.2 mgrm. epinephrine per grm. gland. When 3 units per kilo were dosed, the glands were found to contain a great amount of epinephrine such as $1.3-2.2 \mathrm{mgrm}$. in toto or $0.55-0.081 \mathrm{mgrm}$. epinephrine per grm.; but when the measurement was made a little later, so large a value was never met with. In passing, some figures in the paper of Hiraki, which have no direct bearing upon the present investigations, will be quoted here, in order to complete the reference rê the relation between insulin and the epine-

4) Luttichau, Boll. d. Soc. Ital. di Biol. Sperim., 1927, 2, 133.

5) Hiraki, Kumamoto Igakkai Zassi, 1929, 5, 271 (English abstract p. 30). 
phrine output and the epinephrine load, which are rather completely given in the previous papers ${ }^{1}$ from this Laboratory. On a subcutaneous application of insulin to rabbits the epinephrine load was found to be diminished, as is well known, in his experiments also, but he noted there that the diminution of storage occasioned there was always similar irrespective of when the investigation was made, that is $10,30,60,90,120$ or 240 minutes after the injection; a somewhat remarkable fact. That the cutting of the splanchnic nerve prevents the diminution is also noted in his paper.

The lasting increase of the epinephrine storage in the suprarenals on repeated injections of insulin, made for a long period, and the very transitory increase, these two interesting findings quoted somewhat minutely above, led the present writer to carry out his experiments, the results of which will be given in the following pages. That a repetition of the insulin application causes a hypertrophy of the suprarenal gland, especially of its medulla through exertion is highly interesting. If it may be so, one must further question whether other kinds of agents stimulating the epinephrine output will act in a similar manner. That the medulla takes a share in the hypertrophy of the suprarenal body is not yet known, and in fact $\mathrm{O}$ hara noted enormously large accessories in the two rabbits which received insulin injections for four hundred days, which were proved microscopically as not involving any medullary elements.

\section{Expermental.}

In one set of rabbits, 11 in number, the splanchnic nerves of which were from about a half to ten weeks previously sectioned on the left side per laparotomiam, insulin toronto was injected under the skin in doses varying from 0.1-1.5 units per kilo of body weight every day, 2-3 hours after feeding. At each injection the dose was determined from the symptoms seen on the previous day, so that a moderate intoxication was achieved every time. 34 to 38 injections were carried out in 34 to 38 days, and on the day after the last injection the animals were killed by a blow on the neck, and the suprarenals were taken out at once, and the epinephrine therein was measured by the Suto-Kojima method. ${ }^{6)}$

In the second set of rabbits, 15 in number, in which the left splanchnic nerve of each was sectioned per laparotomiam 3 to 14 weeks previously insulin was injected into an ear vein in the dose of 3 units

6) Kojim a, Tohoku J. of Exp. Med., 1928, 10, 281. 


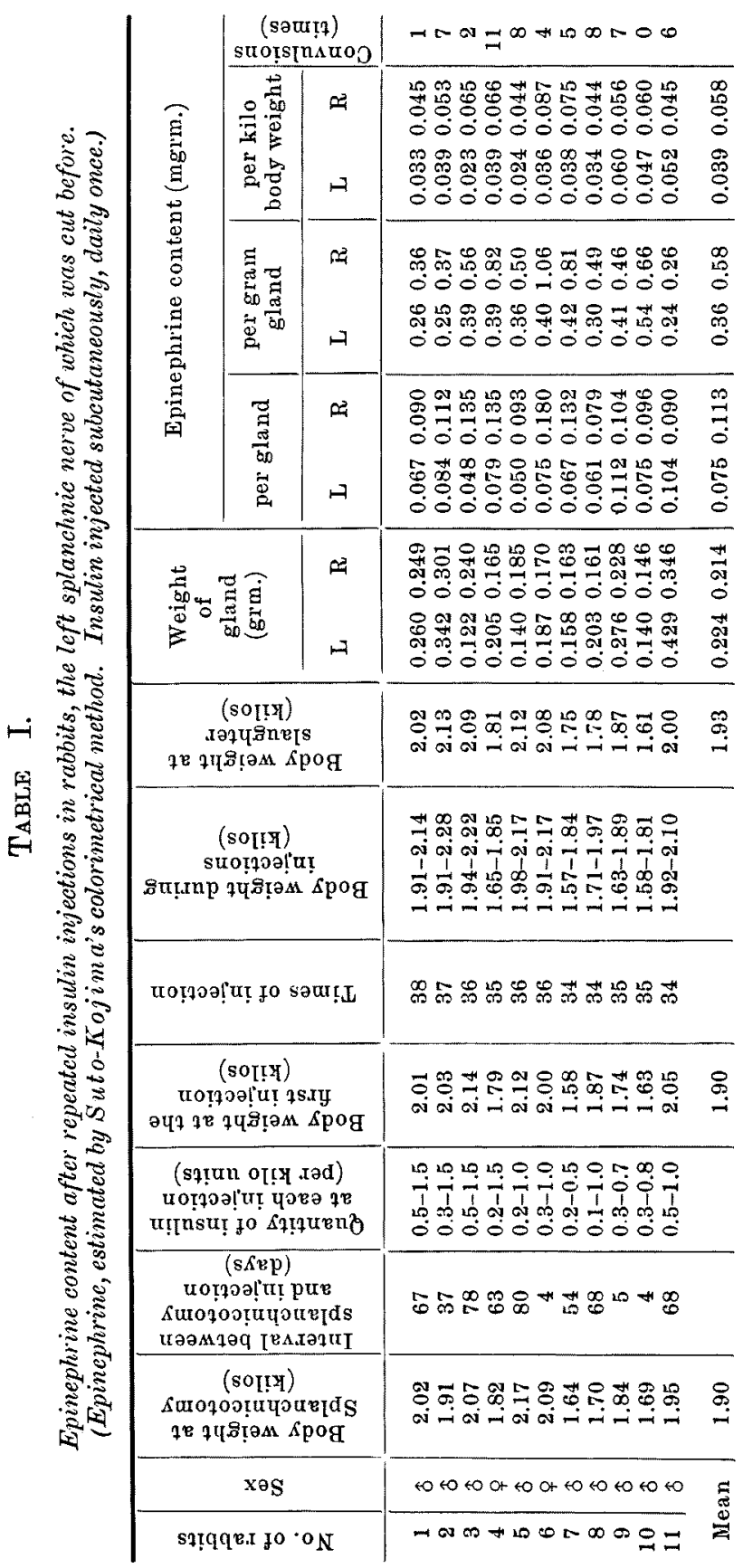


per kilo, and 2 minutes, 4 minutes or 6 minutes later the animal was slaughtered by a blow on the neck, and the suprarenal capsules were worked out.

The results are given in two tables.

In the discussion the standard values are taken from the data of normal rabbits, given in a previous paper ${ }^{i)}$ and from the data of the previous experimentalists, who made use of the same procedure of determining the epinephrine, which are quoted also there.

The data from the rabbits, which received long continued, daily doses of insulin, will be first mentioned.

A few words are added in regard to the body weight; some days after the start of the injection the body weight measured less, but the initial value was almost recovered within about two weeks and afterwards the weight gradually increased over the initial weight. Such an increase of the body weight of rabbits, which receive insulin repeatedly, is due according to $\mathrm{O} h$ a r a to the effect of the insulin. The rabbits ate well.

The rabbits received insulin in doses of 0.1-1.5 units insulin for 34 to 38 days, and were killed 24-30 hours after the last injection; the suprarenals were measured, the left side as $0.122-0.42 \mathrm{grm}$. , mean $0.224 \mathrm{grm}$. and the right side as $0.081-0.343 \mathrm{grm}$., mean $0.214 \mathrm{grm}$. Those of 16 control rabbits, presented in the previous paper? are $\mathrm{L}$ 0.081-0.343 grm., mean $0.182 \mathrm{grm}$. and R 0.069-0.286 grm., mean $0.168 \mathrm{grm}$. The body weight was quite the same in both sets of rabbits, 1.93 kilos and 1.92 kilos respectively. The glands seem to be somewhat enlarged, though not so definitely. ${ }^{87}$

Riddle, Honey well and $\mathrm{Fish}$ is' $\mathrm{r}^{9}$ ) finding in pigeons, that insulin given in a single large dose or repeated less heavy doses enlarges the suprarenal glands, was confirmed by $\mathrm{N} \mathrm{os} \mathrm{aka}^{2}$ working with rats, while $\mathrm{T}$ hatch er ${ }^{10)}$ denied to accept it by the experiments on rabbits. In the rats of Nosaka the weight of the suprarenals in the insulin set was $0.0270 \mathrm{grm}$., that is $0.027 \mathrm{grm}$. per grm. glands, and that in the control, viz. saline solution set was 0.0326 grm., that is $0.025 \mathrm{grm}$. per grm. glands; it is therefore very difficult to speak of a hypertrophy of the suprarenals. The rats received insulin for about two weeks. Of the rabbits of the latter the control series, that is the rabbits treated with the same amount of normal salt solution, had greater weights than the insulintreated series. The injections were continued for several months. Ohara $\mathrm{a}^{3)}$ was able also to see an abundant enlargement of the suprarenals by giving in-

7) Ka now o ka, Tohoku J. of Exp. Med., 1935, 25,

8) Compare: Brown, Pearce and Van Allen, J. of Exp. Med., 1925, 42, 75, Kojima, Tohoku J. of Exp. Med., 1929, 13, 214.

9) Riddle, Honey well and Fisher, Am. J. of Physiol., 1924, 68, 461.

10) Thatcher, J. of Exp. Med., 1926, 43, 357. 
sulin repeatedly, and several times an enormous hypertrophy was noted. The glands, both combined, weighing over 1 grm. were measured in two rabbits treated with insulin for four hundred days; they were a little larger or nearly the same as the maximum weight seen by B row $n$ and others in 350 male rabbits. ${ }^{8)}$ Such a hypertrophy seems to continue after discontinuing the insulin administration, judging from the two casas (Nos. 19 and 20) which were killed about twenty days after the last insulin injection and found to have suprarenals with a weight of 0.7 and 0.8 grm. respectively.

In the present investigations the left gland was previously denervated. In 5 instances out of 11 , that gland was heavier than the right gland; in two cases both glands had similar weight. Such a difference is however physiological in rabbits. In the control set, presented in the previous paper, ${ }^{7}$ ) the left gland was heavier than its fellow in 11 cases out of 16 , and in two cases the reverse held true. Splanchnicotomy has thus no visible influence upon the suprarenal weight of rabbits, treated with insulin for a long period.

The epinephrine content, determined by the colorimetrical method of Suto-Kojima, was found in the left gland, which was denervated beforehand, as $0.048-0.112 \mathrm{mgrm}$, mean $0.075 \mathrm{mgrm}$. in toto, correspondingly $0.24-0.54 \mathrm{mgrm}$., mean $0.36 \mathrm{mgrm}$. per grm. gland, and in the right gland with intact innervation as $0.079-0.180 \mathrm{mgrm}$, mean $0.113 \mathrm{mgrm}$. in toto, that is $0.26-1.06 \mathrm{mgrm}$., mean $0.58 \mathrm{mgrm}$. per grm. gland. In nine cases out of 11, the right gland had a greater load of epinephrine, while in two cases, Nos. 9 and 11, the reverse held true. The difference was only small in these two cases; and if the content be reckoned on the weight of the gland the denervated gland involved invariably a smaller amount of epinephrine.

The epinephrine amount in the gland with the intact innervation is somewhat larger compared with that in the normal rabbits in my previous report and in the previous investigations, quoted there; and that in the denervated gland is somewhat smaller. But, the amount of epinephrine reckoned on the body weight is decidedly smaller in the denervated gland, while that in the right gland with the intact innervation is wholly normal. Is it then reasonable to assume a reduction of the epinephrine storage by repeated treatments with insulin? If the epinephrine percentage only be taken into consideration, this inference is right, but in the present experiments the suprarenal glands enlarged to a certain degree. It is therefore rather fair to compare the total amount of epinephrine existing in the glands. The mean value of epinephrine in each gland of rabbits is about 0.09 mgr'm. The right gland in the present investigations involved a mean of $0.113 \mathrm{mgrm}$., a somewhat larger amount than the control, normal ones, and the left, denervated gland a mean of $0.075 \mathrm{mgrm}$., a some- 
what smaller quantity. Then has the repeated injection of insulin caused an increase of epinephrine in the gland with the intact innervation and a decrease in the gland deprived of the innervation? Does the gland which is denervated involve the same amount of epinephrine as that with the intact innervation or not? While Hiray a $\mathrm{ma}^{11)}$ is of the opinion that the denervation does not alter the amount of epinephrine in the gland, $\mathrm{Tach}^{12}{ }^{12}$ detected invariably a smaller amount of epinephrine in the denervated gland of rabbits, in harmony with $\mathrm{Fu} \mathrm{jii}^{13)}$ in dogs. In the rabbits of $\mathrm{Tach} i$ the diminution was of about twenty percent. He made use of Kodama's modification of the colorimetrical method of Folin, Cannon and Denis for determining the epinephrine. Hiraki, ${ }^{5)}$ who determined the epinephrine by means of the Suto method, reported that the epinephrine load is reduced in the gland with the intact innervation a few days after the operation, but already recovered within 4 to 5 days after the operation. A long interval was allowed to elapse between the denervation and the epinephrine determination in the experiments of Tachi and others. If the finding of Tachi be granted as applying in the present cases, the values for the left gland can be taken as nearly normal. At present it must be left open, without saying the definiteness, whether a repeated injection of insulin extending for several days acts to reduce the epinephrine storage of the denervated suprarenal capsule or not. Only it is sure that such a treatment causes an increase of the epinephrine load of the gland with intact innervation. This finding coincides with those of Nosaka on rats and of Ohara on rabbits, though the increase in the present investigation was on a much smaller scale. In the cases of Nosaka the total amount of epinephrine was estimated as a little greater in the insulin series than in the saline series, but the percentage value was much greater, as much as $0.75 \mathrm{mgrm}$. per grm. against $0.51 \mathrm{mgrm}$. per grm. The absolute weight of the glands were much greater in the saline series of rats than in the insulin series. The epinephrine content of the insulin rabbits of Ohara was much greater than in normal rabbits, if the actual amounts be compared, but if it is reckoned on the gland weight the insulin rabbits had mostly a similar amount with normal animals. This is due to an enlargement of the suprarenal glands. It is highly probable that the enlargement of the glands at least is

11) Hiray a ma, Tohoku J. of Exp. Med., 1925, 5, 573.

12) T a c h i, Ibid., 1928, 10, 409.

13) F u ji i, Ibid., 1925, 5, 571. 
chiefly due to that of the cortex, but not of the medulla. Ohara noted some hypertrophied cortical tissues in the two rabbits which were poisoned with insulin for four hundred days, and no medullary elements were found in them.

This increase of the epinephrine content may be taken as a token of hyperproduction through repeated excessive work, and it is quite sure that the integrity of the innervation is necessary to increase the epinephrine load. The hypersecretion of epinephrine by insulin from the suprarenal gland depends upon the integrity of the splanchnic nerves (a personal communication of Doctors K. Saidzo and M. Hatano). The sectioning of the splanchnic nerve had no influence upon the enlargement of the glands itself, while it interfers with the overload of epinephrine therein.

Lastly the data from the rabbits which received insulin intravenously will be discussed.

32 to 139 days after the left splanchnicotomy the rabbits received insulin into an ear vein in a dose of 3 units per kilo of body weight, and 2, 4 or 6 minutes thereafter they were killed by a blow on the neck, and the suprarenal capsules were at once examined for the epinephrine content.

The left glands were in general a little heavier than the right ones as usual. In 5 rabbits killed two minutes after the injection, the right gland of four individuals involved more epinephrine than the left denervated gland, while in one rabbit both glands had the same amount of epinephrine. The figures yielded in three cases can be taken as an increase of epinephrine load, by comparing with the normal values. The content in the denervated glands was a little smaller than the control. A large content of the epinephrine was also found in 4 glands of 6 rabbits killed with 4 minutes-interval; one was the denervated gland. A somewhat large content was also noted in a single case among the rabbits killed 6 minutes after insulin.

That insulin acts to reduce the epinephrine storage is a well established fact, but we are now able to see the fact that insulin also induces an increase in the epinephrine load within a few minutes, provided it is given intravenously, a somewhat strange fact detected by Hiraki, although our figures were not so significant as his. And this is not detectable with constancy, as is shown in Hiraki's protocols also. 


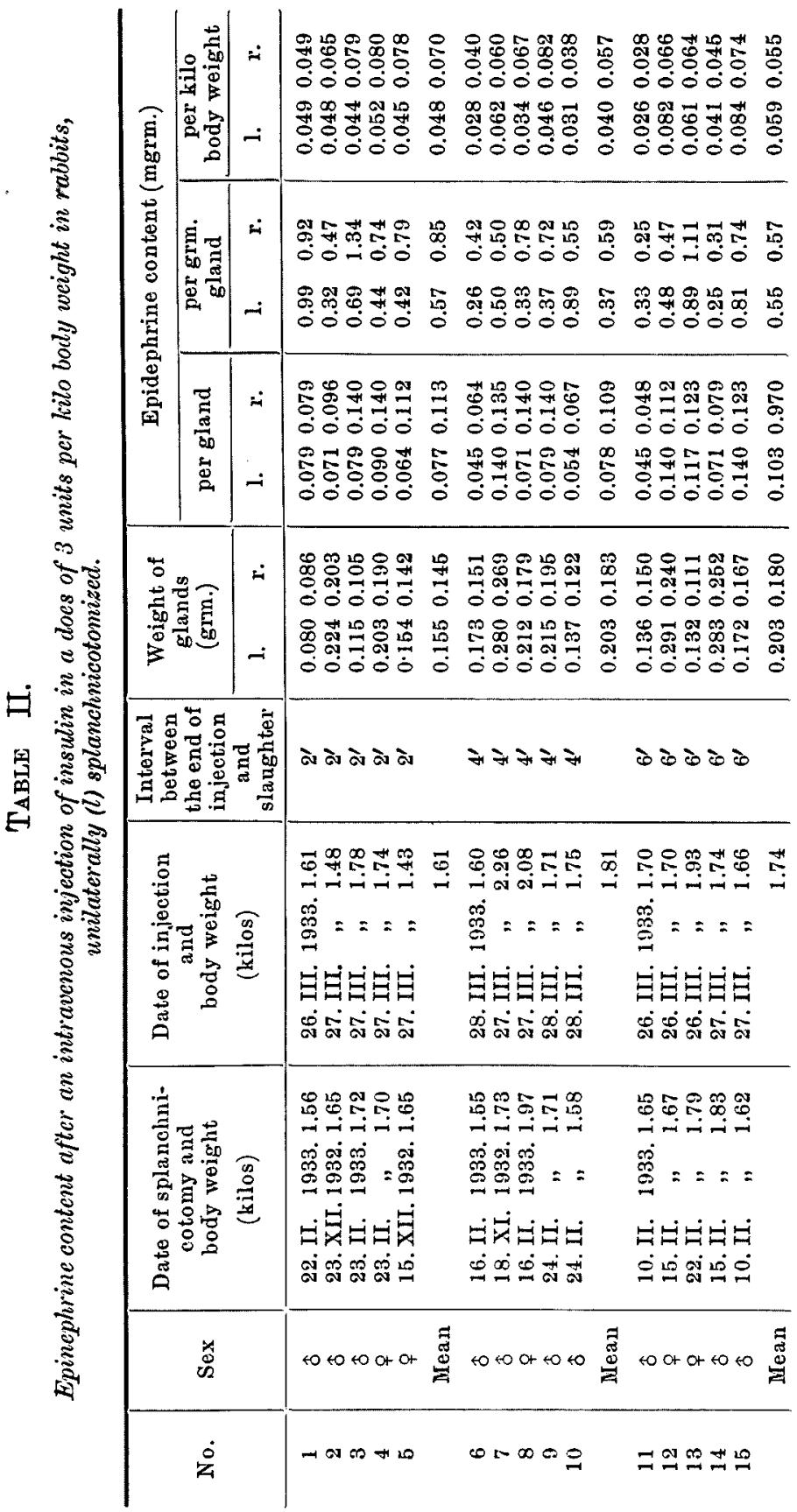




\section{Summary.}

Rabbits received insulin every day in doses of 0.1-1.5 units for about five weeks; $24-30$ hours after the last injection the animals were killed for determining the epinephrine content by means of the SutoKojima's colorimetrical method. The splanchnic nerve was cut per laparotomiam several days before the first injection.

Both glands were found somewhat enlarged, and the gland with the intact innervation as involving a somewhat large amount of epinephrine. If it be reckoned on the gland weight no deviation from the normal was to be noted, but it is reasonable to take the total amount of epinephrine into account. The denervated gland contained a smaller amount of epinephrine compared with the normal ; the present writer has no good grounds to decide whether to range it within the normal limits inherent in the denervated gland or to take it as abnormally smaller, that is as to the action of the repeated insulin administration upon the denervated gland in the long run. At any rate it is sure that the increase of the epinephrine load in the suprarenals due to a repeated insulin application depends upon the integrity of the innervation of the glands.

A previous finding, that an intravenous administration of insulin brings about a transitory increase of the epinephrine load, was confirmed by the present writer, although it is in a much smaller degree. 